\title{
RELATIVELY COMPACT SETS OF HEISENBERG MANIFOLDS
}

\author{
SEBASTIAN BOLDT
}

\begin{abstract}
We give a necessary and sufficient condition for a set of left invariant metrics on a compact Heisenberg manifold to be relatively compact in the corresponding moduli space.
\end{abstract}

\section{INTRODUCTION}

The classic compactness Theorem of Mahler, also called Mahler's Selection Theorem, states that a set of lattices in $\mathbb{R}^{n}$ has compact closure if and only if the volume of each lattice is uniformly bounded from above and the length of a shortest nontrivial vector in each lattice is uniformly bounded from below. The topology on the space of lattices will become clear later. The statement of the theorem remains true if we replace lattices by flat tori. Indeed, there is a bijective correspondence between (isometry classes of) lattices and (isometry classes of) flat tori, under which shortest nontrivial vectors correspond to shortest nontrivial closed curves.

In this note we are concerned with an extension of this theorem to compact Riemannian Heisenberg manifolds. A compact Heisenberg manifold is a compact quotient of the Heisenberg group by a discrete subgroup. These quotients can be characterised as the total spaces of (nontrivial) $S^{1}$-bundles over even-dimensional tori. Given a left invariant metric, they become Riemannian submersions whose base is a flat torus and whose $S^{1}$-fibres are totally geodesic. One might hope that a set of such metrics has compact closure if the condition of Mahler's theorem applies to the base torus and the fibres. Unfortunately, this is not true. One has to bound an additional invariant.

We will now explain the above in greater detail. Let $L$ be a lattice (of full rank) in $\mathbb{R}^{n}$. Associated with $L$ is the flat torus $T=L \backslash \mathbb{R}^{n}$. The lattice $L$ can be recovered from $T$ as the group of deck transformations of the universal Riemannian covering $\mathbb{R}^{n} \rightarrow T$. We can describe $L$ by a basis, which we view as a matrix $G \in \mathrm{GL}(n ; \mathbb{R})$. This basis is of course not unique, but every other basis of $L$ can be obtained from $G$ by a change of basis, which corresponds to multiplication (from the right) by an element of $\operatorname{GL}(n ; \mathbb{Z})$. Since we are only interested in the isometry class of $L$, we view every lattice that we obtain from an orthogonal transformation applied to $L$ as identical to $L$. This means that the isometry class of $L$ (resp. $T)$ is given by the element $\mathrm{O}(n ; \mathbb{R}) \cdot G \cdot \mathrm{GL}(n ; \mathbb{Z}) \in \mathrm{O}(n ; \mathbb{R}) \backslash \mathrm{GL}(n ; \mathbb{R}) / \mathrm{GL}(n ; \mathbb{Z})$. Now note that the quotient $\mathrm{O}(n ; \mathbb{R}) \backslash \mathrm{GL}(n ; \mathbb{R})$ is diffeomorphic to the space $\mathcal{P}_{n}$ of symmetric, positive definite $n \times n$-matrices: $\operatorname{GL}(n ; \mathbb{R})$ acts from the right on $\mathcal{P}_{n}$ via $\mathcal{P}_{n} \times \mathrm{GL}(n ; \mathbb{R}) \ni(Y, H) \mapsto Y[H]={ }^{\mathrm{t}} H Y H \in \mathcal{P}_{n}$. This action is smooth and transitive and the isotropy group of the identity $\operatorname{Id}_{n} \in \mathcal{P}_{n}$ is exactly $\mathrm{O}(n ; \mathbb{R})$, so that we obtain a diffeomorphism $\mathrm{O}(n ; \mathbb{R}) \backslash \mathrm{GL}(n ; \mathbb{R}) \rightarrow \mathcal{P}_{n}$ given by $\mathrm{O}(n ; \mathbb{R}) \cdot G \mapsto{ }^{\mathrm{t}} G G$.

2010 Mathematics Subject Classification. 58D27, 22E25. 
The matrix ${ }^{\mathrm{t}} G G$ is the Gram matrix of the basis $G$ of $L$. The moduli space of lattices in $\mathbb{R}^{n}$ resp. flat $n$-dimensional tori is thus $\mathcal{P}_{n} / \mathrm{GL}(n ; \mathbb{Z})$.

Given $[Y] \in \mathcal{P}_{n} / \mathrm{GL}(n ; \mathbb{Z})$, the volume of the corresponding torus $T$ is given by $\operatorname{det}(Y)^{1 / 2}$ and the squared length of a shortest nontrivial closed curve by $m(Y)=$ $\inf \left\{Y[a]={ }^{\mathrm{t}} a Y a \mid \in \mathbb{Z} \backslash\{0\}\right\}$. Mahler's compactness Theorem (see Corollary 3.7) states that a set $M \subset \mathcal{P}_{n} / \mathrm{GL}(n ; \mathbb{Z})$ has compact closure if and only if there are constants $C_{0}, C_{1}>0$ such that $\operatorname{det}(Y) \leq C_{1}$ and $m(Y) \geq C_{0}$ for all $[Y] \in M$.

In this note we prove an analogous result for the moduli space of compact Riemannian Heisenberg manifolds. Let $H_{n}$ be the $(2 n+1)$-dimensional Heisenberg group and fix a cocompact discrete subgroup $\Gamma \subset H_{n}$. A Riemannian metric $\mathbf{m}$ on $\Gamma \backslash H_{n}$ is called left invariant if its lift to $H_{n}$ is left invariant, i.e., invariant under left multiplication by $H_{n}$ on itself. The moduli space of such Riemannian metrics is essentially $\mathcal{P}_{2 n} / \widetilde{\mathrm{Sp}}(2 n ; \mathbb{Z}) \times(0, \infty)$ (see Theorem 2.1 for the exact definition). Here, $\widetilde{\mathrm{Sp}}(2 n ; \mathbb{Z})$ is the space of all matrices $A \in \mathrm{GL}(2 n ; \mathbb{R})$ such that ${ }^{\mathrm{t}} A J A= \pm J$, where $J$ is the matrix representation of the standard almost complex structure $J$ on $\mathbb{R}^{2 n}$. We know what the compact subsets of $(0, \infty)$ are. Example 3.9 shows that Mahler's Selection Theorem does not hold for $\mathcal{P}_{2 n} / \widetilde{\mathrm{Sp}}(2 n ; \mathbb{Z})$, i.e., there is a sequence $\left\{\left[Y_{k}\right]\right\}_{k \in \mathbb{N}}$ which does not contain a converging subsequence, yet $m\left(Y_{k}\right)=\operatorname{det}\left(Y_{k}\right)=1$ for all $k$.

The solution is to place a uniform bound on the (absolut value of the) eigenvalues of $Y^{-1} J$. Accordingly, our main result, Theorem 3.13, states the following.

Main Theorem. Let $M \subset \mathcal{P}_{2 n} / \widetilde{\mathrm{Sp}}(2 n ; \mathbb{Z})$. Assume there exist positive constants $C_{0}, C_{1}, C_{2}$ such that $m(Y) \geq C_{0}$, $\operatorname{det}(Y) \leq C_{1}$ and the eigenvalues of $Y^{-1} J$ are bounded in absolute value from above by $C_{2}$ for all $[Y] \in M$. Then $M$ has compact closure.

Necessity of the stated condition follows from the continuity of det, $m$ and the eigenvalues of $Y^{-1} J$.

The proof uses the following idea. Since $\widetilde{\mathrm{Sp}}(2 n ; \mathbb{Z}) \subseteq \mathrm{GL}(2 n ; \mathbb{Z})$ is a subgroup of infinite index $(n>1)$, a fundamental domain for $\mathcal{P}_{2 n} / \widetilde{\mathrm{Sp}}(2 n ; \mathbb{Z})$ could be obtained by gluing an infinite number of displacements of a fundamental domain for $\mathcal{P}_{2 n} / \mathrm{GL}(2 n ; \mathbb{Z})$. Such a domain is Minkowski's fundamental domain, for which the Hermite-Mahler Compactness Theorem (see Theorem 3.6) characterises the compact sets. We then show that the preimage in $\mathcal{P}_{2 n}$ of a set $M \subset \mathcal{P}_{2 n} / \widetilde{\mathrm{Sp}}(2 n ; \mathbb{Z})$ which satisfies the assumptions of Theorem 3.13 is necessarily contained in a finite number of displacements of Minkowski's fundamental domain and satisfies the assumptions of the Hermite-Mahler Theorem and must therefore be compact. The crucial matrix inequality needed in the proof is established in Lemma 3.12.

In geometric terms this means that a set of left invariant metrics on a fixed quotient $\Gamma \backslash H_{n}$ is precompact iff the volume of the $S^{1}$-fibres is bounded from above and below, the systole of the base torus is bounded from below, the volume of the base torus is bounded from above, and that all sectional curvatures are bounded from above by a positive constant, see Remark 3.16 .

The results in this note have been part of the author's PhD-thesis Bol18 and were very recently extended to left invariant sub-Riemannian metrics by K. Tashiro [Tas20]. 
This note is organised as follows. In Section 2 we introduce compact Riemannian Heisenberg manifolds and their moduli spaces. Section 3 contains the main result (Theorem 3.13) and its proof.

\section{ACKNOWLEDGEMENTS}

The author gratefully acknowledges financial support by the Collaborative Research Center 647 - Space - Time - Matter.

\section{Riemannian Heisenberg Manifolds}

This section describes compact Riemannian Heisenberg manifolds. The exposition follows mostly GW86.

For $x, y \in \mathbb{R}^{n}, s \in \mathbb{R}$ we let

$$
\gamma(x, y, s):=\left(\begin{array}{ccc}
1 & { }^{\mathrm{t}} x & s \\
0 & \operatorname{Id}_{n} & y \\
0 & 0 & 1
\end{array}\right), \quad X(x, y, s):=\left(\begin{array}{ccc}
0 & { }^{\mathrm{t}} x & s \\
0 & 0 & y \\
0 & 0 & 0
\end{array}\right) .
$$

The $(2 n+1)$-dimensional Heisenberg group $H_{n}$ is $H_{n}:=\left\{\gamma(x, y, s) \mid x, y \in \mathbb{R}^{n}, s \in \mathbb{R}\right\}$ with the Lie group structure that it inherits as a closed subset of $\operatorname{GL}(n+2 ; \mathbb{R})$. Its Lie algebra is $\mathfrak{h}_{n}=\left\{X(x, y, s) \mid x, y \in \mathbb{R}^{n}, s \in \mathbb{R}\right\}$. The standard basis $\mathfrak{B}_{n}$ of $\mathfrak{h}_{n}$ is

$$
\mathfrak{B}_{n}:=\left(X_{1}, \ldots, X_{n}, Y_{1}, \ldots, Y_{n}, Z\right)
$$

with

$$
\begin{aligned}
X_{j} & :=X\left(e_{j}, 0,0\right) \text { for all } 1 \leq j \leq n, \\
Y_{j} & :=X\left(0, e_{j}, 0\right) \text { for all } 1 \leq j \leq n, \\
Z & :=X(0,0,1),
\end{aligned}
$$

where $\left(e_{1}, \ldots, e_{n}\right)$ is the standard basis of $\mathbb{R}^{n}$. Multiplication and inversion in $H_{n}$ obey the rules

$$
\begin{aligned}
\gamma(x, y, s) \cdot \gamma\left(x^{\prime}, y^{\prime}, s^{\prime}\right) & =\gamma\left(x+x^{\prime}, y+y^{\prime}, s+s^{\prime}+\left\langle x, y^{\prime}\right\rangle\right) \\
\gamma(x, y, s)^{-1} & =\gamma(-x,-y,-s+\langle x, y\rangle)
\end{aligned}
$$

where $\langle\cdot, \cdot\rangle$ denotes the Euclidean inner product. The Lie exponential map $\exp : \mathfrak{h}_{n} \rightarrow H_{n}$

$$
\begin{aligned}
\exp X(x, y, s) & =\operatorname{Id}+X(x, y, s)+\frac{1}{2} X(x, y, s)^{2} \\
& =\gamma\left(x, y, s+\frac{1}{2}\langle x, y\rangle\right),
\end{aligned}
$$

is a global diffeomorphism with inverse $\log : H_{n} \rightarrow \mathfrak{h}_{n}$,

$$
\log \gamma(x, y, s)=X\left(x, y, s-\frac{1}{2}\langle x, y\rangle\right) .
$$

From (2.1) it follows that commutators in $H_{n}$ and $\mathfrak{h}_{n}$ are given by

$$
\begin{aligned}
{\left[\gamma(x, y, s), \gamma\left(x^{\prime}, y^{\prime}, s^{\prime}\right)\right] } & =\gamma\left(0,0, A\left((x, y),\left(x^{\prime}, y^{\prime}\right)\right)\right), \\
{\left[X(x, y, s), X\left(x^{\prime}, y^{\prime}, s^{\prime}\right)\right] } & =X\left(0,0, A\left((x, y),\left(x^{\prime}, y^{\prime}\right)\right)\right),
\end{aligned}
$$

where $A$ is the standard symplectic form on $\mathbb{R}^{2 n}$ whose matrix resepresentation w.r.t. the standard basis is

$$
J=\left(\begin{array}{cc}
0 & \mathrm{Id}_{n} \\
-\mathrm{Id}_{n} & 0
\end{array}\right)
$$


Since $A$ is nondegenerate it follows from (2.2) that the centre $\mathfrak{z}=\mathfrak{z}_{n}$ of $\mathfrak{h}_{n}$ is $\mathfrak{z}_{n}=\{X(0,0, s) \mid s \in \mathbb{R}\}$. We identify the subspace $\left\{X(x, y, 0) \mid(x, y) \in \mathbb{R}^{2 n}\right\}$ with $\mathbb{R}^{2 n}$. Under this identification $\mathfrak{h}_{n}$ is the direct sum $\mathfrak{h}_{n}=\mathbb{R}^{2 n} \bigoplus \mathfrak{z}$ and $\left(X_{1}, \ldots, X_{n}, Y_{1}, \ldots, Y_{n}\right)$ is the standard basis of $\mathbb{R}^{2 n}$. By (2.2) we have for all $X, Y \in \mathbb{R}^{2 n}$

$$
[X, Y]=A(X, Y) Z \text {. }
$$

We now turn to the automorphisms of $H_{n}$ and $\mathfrak{h}_{n}$. Since $H_{n}$ is connected and by the naturality of the Lie exponential map, we will identify automorphisms of $H_{n}$ with their differentials at the identity. These are precisely the automorphisms of $\mathfrak{h}_{n}$. Furthermore, we will identify an automorphism $\varphi \in \operatorname{Aut}\left(\mathfrak{h}_{n}\right)$ with its matrix representation relative to the basis $\mathfrak{B}_{n}$. Let

$$
\widetilde{\mathrm{Sp}}(2 n ; \mathbb{R}):=\left\{\left.\beta \in \mathrm{GL}(2 n ; \mathbb{R})\right|^{\mathrm{t}} \beta J \beta=\epsilon(\beta) J, \varepsilon(\beta)= \pm 1\right\},
$$

with $J$ as in (2.3). We imbed $\widetilde{\mathrm{Sp}}(2 n ; \mathbb{R})$ into $\operatorname{GL}(2 n+1 ; \mathbb{R})$ via

$$
\widetilde{\mathrm{Sp}}(2 n ; \mathbb{R}) \ni \beta \mapsto\left(\begin{array}{cc}
\beta & 0 \\
0 & \epsilon(\beta)
\end{array}\right) \in \mathrm{GL}(2 n+1 ; \mathbb{R}) .
$$

For $a \in \mathbb{R} \backslash\{0\}$ and $w \in \mathbb{R}^{2 n}$, let

$$
\alpha(a, w):=\left(\begin{array}{cc}
a \cdot \operatorname{Id}_{2 n} & 0 \\
w^{t} & a^{2}
\end{array}\right) \in \mathrm{GL}(2 n+1 ; \mathbb{R}) .
$$

Simple calculations show that the group Aut $\left(\mathfrak{h}_{n}\right)$ consists of all products of the form $\alpha(a, w) \cdot \beta, a \in \mathbb{R} \backslash\{0\}, w \in \mathbb{R}^{2 n}, \beta \in \widetilde{\mathrm{Sp}}(2 n ; \mathbb{R})$. The inner automorphisms are those for which $a=1$ and $\beta=\operatorname{Id}_{2 n}$.

We introduce a set of distinguished uniform (i.e., discrete and cocompact) subgroups of $H_{n}$. Let

$$
\mathcal{D}_{n}:=\left\{r=\left(r_{1}, \ldots, r_{n}\right) \in \mathbb{N}^{n}\left|\forall i \in\{1, \ldots, n-1\}: r_{i}\right| r_{i+1}\right\} .
$$

For an $r \in \mathcal{D}_{n}$ define the matrix

$$
\delta_{r}:=\operatorname{diag}\left(r_{1}, \ldots, r_{n}, 1, \ldots, 1\right)
$$

and the uniform subgroup $\Gamma^{r} \subset H_{n}$ by

$$
\Gamma^{r}:=\left\{\gamma(x, y, s) \mid\left(\begin{array}{l}
x \\
y
\end{array}\right) \in \delta_{r} \cdot \mathbb{Z}^{2 n}, s \in \mathbb{Z}\right\} .
$$

We now address the Riemannian structure of Heisenberg manifolds. We call any Riemannian metric $\mathbf{m}$ on a compact Heisenberg manifold $\Gamma \backslash H_{n}$ that originates from a left invariant metric on $H_{n}$ left invariant. Any such metric is uniquely determined by its values at the (equivalence class of the) identity, i.e., by the induced inner product on $\mathfrak{h}_{n}$. Via the basis $\mathfrak{B}_{n}$, we identify the space $\mathscr{M}\left(\mathfrak{h}_{n}\right)$ of inner products on $\mathfrak{h}_{n}$ with $\mathcal{P}_{2 n+1}$, the space of symmetric positive definite $(2 n+1) \times(2 n+1)$-matrices. In case that for a given metric $\mathbf{m} \in \mathcal{P}_{2 n+1}$ the subspaces $\mathfrak{z} \subset \mathfrak{h}_{n}$ and $\mathbb{R}^{2 n} \subset \mathfrak{h}_{n}$ are orthogonal, the metric takes the form

$$
\mathbf{m}=\left(\begin{array}{ll}
h & 0 \\
0 & g
\end{array}\right) \in \mathcal{P}_{2 n} \times(0, \infty) .
$$

We call such a metric normalised and write $\mathbf{m}=(h, g)$. We also call a compact Riemannian Heisenberg manifold $\left(\Gamma^{r} \backslash H_{n}, \mathbf{m}\right)$, where $\mathbf{m}=(h, g)$ is a normalised metric, a normalised Heisenberg manifold.

If $\mathbf{m}=(h, g)$ is a normalised metric and $\alpha(a, w) \cdot \beta \in \operatorname{Aut}\left(\mathfrak{h}_{n}\right)$, then $(\alpha(a, w)$. $\beta)^{*} \mathbf{m}$ is a normalised metric if and only if $w=0$. Note that w.r.t. our identifications, 
the pullback of a metric $\mathbf{m}$ by an automorhism $\varphi$ corresponds to (the restriction of) the action of $\mathrm{GL}(2 n+1 ; \mathbb{R})$ on $\mathcal{P}_{2 n+1}: \varphi^{*} \mathbf{m}=\mathbf{m}[\varphi]={ }^{\mathrm{t}} \varphi \mathbf{m} \varphi$.

Furthermore, for $r \in \mathcal{D}_{n}$ we have $\alpha(a, 0) \cdot \beta \log \Gamma^{r}=\log \Gamma^{r}$ if and only if $a=1$ and $\beta \in \delta_{r} \mathrm{GL}(2 n ; \mathbb{Z}) \delta_{r}^{-1}$. We therefore define

$$
G_{r}:=\delta_{r} \mathrm{GL}(2 n ; \mathbb{Z}) \delta_{r}^{-1}, \quad \Pi_{r}:=G_{r} \cap \widetilde{\mathrm{Sp}}(2 n ; \mathbb{R}) .
$$

Theorem 2.1 (GW86, Corollary 2.5, Theorem 2.7]). Every compact Riemannian Heisenberg manifold is isometric to a normalised Heisenberg manifold. Moreover, two normalised Heisenberg manifolds $\left(\Gamma^{r} \backslash H_{n}, \mathbf{m}\right)$ and $\left(\Gamma^{s} \backslash H_{n}, \mathbf{m}^{\prime}\right)$ are isometric iff $r=s$ and $\mathbf{m}^{\prime}=\beta^{*} \mathbf{m}$ for some $\beta \in \Pi_{r}$. They are homeomorphic iff $r=s$. Accordingly, the set

$$
\mathcal{M}_{n}:=\bigcup_{r \in \mathcal{D}_{n}} \mathcal{M}_{n}^{r}, \quad \mathcal{M}_{n}^{r}:=\left(\mathcal{P}_{2 n} \times(0, \infty)\right) / \Pi_{r}
$$

parametrises the isometry classes of compact Riemannian Heisenberg manifolds.

The Kaplan map $j: \mathfrak{z} \rightarrow \mathfrak{s o}\left(\mathbb{R}^{2 n}, h\right)$ of a normalised Heisenberg manifold $\left(\Gamma^{r} \backslash H_{n}, \mathbf{m}\right), \mathbf{m}=(h, g)$, is defined by

$$
\langle j(W) X, Y\rangle_{\mathbf{m}}=\langle W,[X, Y]\rangle_{\mathbf{m}},
$$

for all $W \in \mathfrak{z}, X, Y \in \mathbb{R}^{2 n}$. Here, $\mathfrak{s o}\left(\mathbb{R}^{2 n}, h\right)$ denotes the space of skew-symmetric endomorphisms of $\left(\mathbb{R}^{2 n}, h\right)$ which we identify via the standard basis with the $2 n \times 2 n$ matrices which are skew-symmetric w.r.t. $h$. From (2.4) it follows that

$$
j\left(g^{-1 / 2} Z\right)=-g^{1 / 2} h^{-1} J .
$$

The normalised Heisenberg manifold $\left(\Gamma^{r} \backslash H_{n}, \mathbf{m}\right)$ is of Heisenberg type if the eigenvalues of $j\left(g^{-1 / 2} Z\right)$ are $\pm i$, i.e., if the eigenvalues of $h^{-1} J$ are $\pm i g^{-1 / 2}$.

At last, we mention the sectional curvatures of $\left(\Gamma^{r} \backslash H_{n}, \mathbf{m}\right)$ which can be easily expressed using the Kaplan map. Let $X, Y \in \mathbb{R}^{2 n}$ be orthonormal elements of $\left(\mathfrak{h}_{n}, \mathbf{m}\right)$. Then the sectional curvature $K$ of $\left(\Gamma^{r} \backslash H_{n}, \mathbf{m}\right)$ is given by

$$
\begin{aligned}
& K(X, Y)=-\frac{3}{4}|[X, Y]|_{\mathbf{m}}^{2}, \\
& K(X, Z)=\frac{1}{4}\left|j\left(g^{-1 / 2} Z\right) X\right|_{\mathbf{m}}^{2},
\end{aligned}
$$

see [Ebe94, (2.4)] (note the wrong sign on the right hand side of (2.4) b) though).

\section{Relatively Compact Sets of Riemannian Heisenberg Manifolds}

Let $Y \in \mathcal{P}_{2 n}$. From (2.4) and (2.5) we know that the map $Y^{-1} J$ is skewsymmetric w.r.t. the inner product on $\mathbb{R}^{2 n}$ defined by $Y$. Thus the eigenvalues of $Y^{-1} J$ are purely imaginary and come in complex conjugate pairs.

\section{Definition and Remarks 3.1.}

(i) For every $n \in \mathbb{N}$ we define $n$ functions $d_{1}, \ldots, d_{n}: \mathcal{P}_{2 n} \rightarrow(0, \infty)$ such that $\pm i d_{k}(Y), k=1, \ldots, n$, are the eigenvalues of $Y^{-1} J$ and $d_{1} \leq d_{2} \leq \ldots \leq d_{n}$.

(ii) Note that the $d_{k}$ are continuous (see, e.g., [Zed65, Theorem 1]).

(iii) The functions $d_{1}, \ldots, d_{n}: \mathcal{P}_{2 n} \rightarrow(0, \infty)$ are invariant under the action of $\widetilde{\mathrm{Sp}}(2 n ; \mathbb{R})$. Indeed, for $Y \in \mathcal{P}_{2 n}$ and $A \in \widetilde{S p}(2 n ; \mathbb{R})$ one has

$$
\begin{aligned}
(Y[A])^{-1} J & =\left({ }^{\mathrm{t}} A Y A\right)^{-1} J=A^{-1} Y^{-1 \mathrm{t}} A^{-1} J \sim A\left(A^{-1} Y^{-1 \mathrm{t}} A^{-1} J\right) A^{-1} \\
& =Y^{-1 \mathrm{t}} A^{-1} J A^{-1}=\varepsilon(A) Y^{-1} J
\end{aligned}
$$


with $\varepsilon(A)= \pm 1$. By definition of the $d_{k}$ we thus have $d_{k}(Y[A])=d_{k}(Y)$ for all $1 \leq k \leq n, Y \in \mathcal{P}_{2 n}$ and $A \in \widetilde{\operatorname{Sp}}(2 n ; \mathbb{R})$.

(iv) Define $\mathcal{P}_{2 n}^{*}(Y):=\{Y[S] \mid S \in \widetilde{\mathrm{Sp}}(2 n ; \mathbb{R})\}=\left\{{ }^{\mathrm{t}} S Y S \mid S \in \widetilde{\mathrm{Sp}}(2 n ; \mathbb{R})\right\}$.

Proposition 3.2. Let $X, Y \in \mathcal{P}_{2 n}$. Then

$$
X \in \mathcal{P}_{2 n}^{*}(Y) \quad \text { iff } \quad Y \in \mathcal{P}_{2 n}^{*}(X) \quad \text { iff } \quad d_{j}(X)=d_{j}(Y) \text { for all } 1 \leq j \leq n .
$$

Proof. The first 'iff' is a direct consequence of the definition of $\mathcal{P}_{2 n}^{*}(M), M \in \mathcal{P}_{2 n}$.

We first prove the second 'if': By assumption $X^{-1} J \sim Y^{-1} J$. Since $X^{-1} J \sim$ $X^{-1 / 2} J X^{-1 / 2}$, we have $X^{-1 / 2} J X^{-1 / 2} \sim Y^{-1 / 2} J Y^{-1 / 2}$, which means that there is $A \in \mathrm{O}(2 n ; \mathbb{R})$ such that

$$
{ }^{\mathrm{t}} A X^{-1 / 2} J X^{-1 / 2} A=Y^{-1 / 2} J Y^{-1 / 2} .
$$

This implies that $X^{-1 / 2} A Y^{1 / 2}=: S \in \widetilde{\mathrm{Sp}}(2 n ; \mathbb{R})$. By definition of $S$ we have $A Y^{1 / 2}=$ $X^{1 / 2} S$ and hence $Y=Y^{1 / 2}{ }^{\mathrm{t}} A A Y^{1 / 2}=\operatorname{Id}\left[A Y^{1 / 2}\right]=\operatorname{Id}\left[X^{1 / 2} S\right]={ }^{\mathrm{t}} S X S$ as claimed. The 'only if' part is proved as follows: If $Y \in \mathcal{P}_{2 n}^{*}(X)$ then $Y={ }^{\mathrm{t}} S X S$ for some $S \in \widetilde{\mathrm{Sp}}(2 n ; \mathbb{R})$. But then $Y^{-1} J=S^{-1} X^{-1}{ }^{\mathrm{t}} S^{-1} J= \pm S^{-1} X^{-1} J S \sim \pm X^{-1} J$ since $S \in \widetilde{\mathrm{Sp}}(2 n ; \mathbb{R})$.

Theorem 2.1 now takes the following form for compact Heisenberg manifolds of Heisenberg type.

Corollary 3.3. The normalised Heisenberg manifold $\left(\Gamma^{r} \backslash H_{n}, \mathbf{m}\right)$ with $\mathbf{m}=(h, g)$ is of Heisenberg type if and only if $h \in \mathcal{P}_{2 n}^{*}\left(g^{1 / 2} \mathrm{Id}\right)$ if and only if $d_{k}(h)=g^{-1 / 2}$ for all $1 \leq k \leq n$. Accordingly, the set

$$
\mathcal{M}_{n}^{H T}:=\bigcup_{r \in \mathcal{D}_{n}} \mathcal{M}_{n}^{r, H T}, \quad \mathcal{M}_{n}^{r, H T}:=\left\{[(h, g)] \in \mathcal{M}_{n}^{r} \mid d_{k}(h)=g^{-1 / 2}, 1 \leq k \leq n\right\}
$$

parametrises the isometry classes of normalised Heisenberg manifolds of Heisenberg type. Moreover, each $\mathcal{M}_{n}^{r, H T}$ is closed in $\mathcal{M}_{n}^{r}$.

Proof. By the last proposition $\left(\Gamma^{r} \backslash H_{n}, \mathbf{m}\right)$ is of Heisenberg type if and only if $d_{k}(h)=g^{-1 / 2}$ for all $1 \leq k \leq n$. This, in turn, is the case if and only if $h \in \mathcal{P}_{2 n}^{*}\left(g^{1 / 2} \mathrm{Id}\right)$. It follows from Theorem 2.1 that $\mathcal{M}_{n}^{H T}$ is a moduli space for normalised Heisenberg manifolds of Heisenberg type. The set $\mathcal{M}_{n}^{r, H T}$ is closed in $\mathcal{M}_{n}^{r}$ because the $d_{k}$ are continuous.

We will now study the compact sets of the moduli space $\mathcal{M}_{n}^{r}$.

\section{Notation and Remarks 3.4.}

(i) Minkowski's fundamental domain in $\mathcal{P}_{n}$ is the domain

$$
\begin{aligned}
& \mathscr{M}_{n}=\left\{Y=\left(y_{i, j}\right) \in \mathcal{P}_{n} \mid \forall k=1, \ldots, n: y_{k, k+1} \geq 0\right. \\
&\text { and } \left.Y[a] \geq y_{k, k} \text { for all } a \in \mathbb{Z}^{n} \text { with } \operatorname{gcd}\left(a_{k}, \ldots, a_{n}\right)=1\right\} .
\end{aligned}
$$

By Ter88, CH. IV, Thm. 1], $\mathscr{M}_{n}$ is a connected and closed fundamental domain for $\mathcal{P}_{n} / \mathrm{GL}(n ; \mathbb{R})$.

(ii) For $Y \in \mathcal{P}_{n}$ we define

$$
m(Y):=\inf \left\{Y[a] \mid a \in \mathbb{Z}^{n} \backslash\{0\}\right\} .
$$


The value $m(Y)$ is called the first minimum of $Y$. It is the squared norm of a shortest nonzero vector of a lattice with Gram matrix $Y$. Note that $m(Y)=y_{1,1}$ for $Y \in \mathscr{M}_{n}$ by the very definition of $\mathscr{M}_{n}$.

(iii) For $r \in \mathcal{D}_{n}$ and $Y \in \mathcal{P}_{2 n}$ we define

$$
m_{r}(Y):=\inf \left\{Y\left[\delta_{r} a\right] \mid a \in \mathbb{Z}^{2 n} \backslash\{0\}\right\}=m\left(Y\left[\delta_{r}\right]\right) .
$$

If $r=(1, \ldots, 1)$, we abbreviate $m_{r}(Y)$ simply to $m(Y)$ which is in accordance with (ii).

(iv) The function $m: \mathcal{P}_{n} \rightarrow(0, \infty)$ is constant on the orbits of the action of $\mathrm{GL}(n ; \mathbb{Z})$ on $\mathcal{P}_{n}$ and we denote the induced function on $\mathcal{P}_{n} / \mathrm{GL}(n ; \mathbb{Z})$ by $m$, too.

Similarly, the function $m_{r}: \mathcal{P}_{2 n} \rightarrow(0, \infty)$ is constant on the orbits of the action of $G_{r}=\delta_{r} \mathrm{GL}(2 n ; \mathbb{Z}) \delta_{r}^{-1}$ on $\mathcal{P}_{2 n}$ and we denote the induced function on the quotient by $m_{r}$ as well.

Remark 3.5. For $r \in \mathcal{D}_{n}$, define the map

$$
\begin{aligned}
\Psi_{r}: \mathcal{P}_{2 n} & \rightarrow \mathcal{P}_{2 n} \\
Y & \mapsto Y\left[\delta_{r}^{-1}\right]=\delta_{r}^{-1} Y \delta_{r}^{-1} .
\end{aligned}
$$

Then $\Psi_{r}$ induces a map

$$
\begin{aligned}
\psi_{r}: \mathcal{P}_{2 n} / \mathrm{GL}(2 n ; \mathbb{Z}) & \rightarrow \mathcal{P}_{2 n} / G_{r} \\
{[Y] } & \mapsto\left[Y\left[\delta_{r}^{-1}\right]\right]=\left[\delta_{r}^{-1} Y \delta_{r}^{-1}\right] .
\end{aligned}
$$

The maps $\Psi_{r}$ and $\psi_{r}$ are diffeomorphisms and satisfy $\pi_{r} \circ \Psi_{r}=\psi_{r} \circ \pi$ where $\pi: \mathcal{P}_{2 n} \rightarrow \mathcal{P}_{2 n} / \mathrm{GL}(2 n ; \mathbb{Z})$ and $\pi_{r}: \mathcal{P}_{2 n} \rightarrow \mathcal{P}_{2 n} / G_{r}$ are the canonical projections. It follows that

$$
\mathscr{M}_{2 n, r}:=\Psi_{r}\left(\mathscr{M}_{2 n}\right)
$$

is a fundamental domain for the space $\mathcal{P}_{2 n} / G_{r}$. Note that $m_{r}\left(\Psi_{r}(Y)\right)=m(Y)$ and $m_{r}\left(\psi_{r}([Y])\right)=m([Y])$.

The following classic theorem characterises the relatively compact subsets of $\mathscr{M}_{n}$ and is attributed to Hermite and Mahler by A. Terras.

Theorem 3.6 (Hermite-Mahler Compactness Theorem, see [Ter88, CH. 4.4, Ex. 13]). Any set $M \subset \mathscr{M}_{n}$ for which there are positive constants $C_{0}, C_{1}>0$ such that $m(Y) \geq C_{0}$ and $\operatorname{det} Y \leq C_{1}$ for all $Y \in M$ has compact closure in $\mathscr{M}_{n}$.

From this one easily deduces Mahler's compactness theorem in its original form, which we include for the sake of completeness.

Corollary 3.7 (Selection Theorem of Mahler, cf. GL87, Ch. $3 \S 17$ Theorem 2]). Let $M \subset \mathcal{P}_{n} / \mathrm{GL}(n ; \mathbb{Z})$ be a set such that there are constants $C_{0}, C_{1}>0$ so that $m([Y]) \geq C_{0}$ and $\operatorname{det}([Y]) \leq C_{1}$ for all $[Y] \in M$. Then $M$ has compact closure in $\mathcal{P}_{n} / \mathrm{GL}(n ; \mathbb{Z})$.

Corollary 3.8. Let $n \in \mathbb{N}, r \in \mathcal{D}_{n}$ and $M \subset \mathcal{P}_{2 n} / G_{r}$ be a set such that there are constants $C_{0}, C_{1}>0$ so that $m_{r}([Y]) \geq C_{0}$ and $\operatorname{det}([Y]) \leq C_{1}$ for all $[Y] \in M$. Then $M$ has compact closure in $\mathcal{P}_{2 n} / G_{r}$.

Proof. This follows from the last corollary via the diffeomorphism $\psi_{r}$ defined in Remark 3.5. 
The following example shows that Corollary 3.8 does not remain true if we replace $\mathcal{P}_{2 n} / G_{r}$ by $\mathcal{P}_{2 n} / \Pi_{r}$.

Example 3.9. Let $k \in \mathbb{N}_{0}$ and

$$
Y_{k}:=\left(\begin{array}{cccc}
1 & k & 0 & 0 \\
k & k^{2}+1 & 0 & 0 \\
0 & 0 & 1 & 0 \\
0 & 0 & 0 & 1
\end{array}\right)=\left(\begin{array}{cccc}
1 & k & 0 & 0 \\
0 & 1 & 0 & 0 \\
0 & 0 & 1 & 0 \\
0 & 0 & 0 & 1
\end{array}\right) \cdot \mathrm{Id} \cdot\left(\begin{array}{cccc}
1 & k & 0 & 0 \\
0 & 1 & 0 & 0 \\
0 & 0 & 1 & 0 \\
0 & 0 & 0 & 1
\end{array}\right) .
$$

Obviously, $\operatorname{det}\left(Y_{k}\right)=1$ for all $k \in \mathbb{N}_{0}$. Also, since $Y_{k}$ is in the same $\operatorname{GL}(4 ; \mathbb{Z})$-orbit as Id, $m\left(Y_{k}\right)=1$ for all $k \in \mathbb{N}_{0}$. One easily calculates

$$
Y_{k}^{-1} \cdot J=\left(\begin{array}{cccc}
k^{2}+1 & -k & 0 & 0 \\
-k & 1 & 0 & 0 \\
0 & 0 & 1 & 0 \\
0 & 0 & 0 & 1
\end{array}\right) \cdot J=\left(\begin{array}{cccc}
0 & 0 & k^{2}+1 & -k \\
0 & 0 & -k & 1 \\
-1 & 0 & 0 & 0 \\
0 & -1 & 0 & 0
\end{array}\right),
$$

so that

$$
\left(Y_{k}^{-1} \cdot J\right)^{2}=\left(\begin{array}{cccc}
-k^{2}-1 & k & 0 & 0 \\
k & -1 & 0 & 0 \\
0 & 0 & -k^{2}-1 & k \\
0 & 0 & k & -1
\end{array}\right)
$$

The eigenvalues of $\left(Y_{k}^{-1} \cdot J\right)^{2}$ are thus the solutions of

$$
0=\left(\left(X+k^{2}+1\right)(X+1)-k^{2}\right)^{2}=\left(X^{2}+\left(k^{2}+2\right) X+1\right)^{2} .
$$

It follows that

$$
\begin{aligned}
& d_{1}\left(Y_{k}\right)=2^{-1 / 2} \sqrt{k^{2}+2-k \sqrt{k^{2}+4}}, \\
& d_{2}\left(Y_{k}\right)=2^{-1 / 2} \sqrt{k^{2}+2+k \sqrt{k^{2}+4}} .
\end{aligned}
$$

The sequence $d_{2}\left(Y_{k}\right)$ is monotonously and unboundedly increasing in $k$. Since $d_{2}$ is an invariant of the $\widetilde{\mathrm{Sp}}(4 ; \mathbb{R})$-action (see Remarks 3.1(iii)), no two matrices of the family $\left\{Y_{k}\right\}_{k \in \mathbb{N}}$ are in the same $\widetilde{\operatorname{Sp}}(4 ; \mathbb{Z})$-orbit. Note that the $d_{j}(Y), j=1,2$, are continuous in $Y \in \mathcal{P}_{4}$ (see Remarks 3.1(iii)) and descend to continuous functions on $\mathcal{P}_{4} / \widetilde{\mathrm{Sp}}(4 ; \mathbb{Z})$. Therefore, no subsequence of $\left\{\left[Y_{k}\right]\right\}_{k \in \mathbb{N}}$ converges. In particular, boundedness of $\operatorname{det}(\cdot)$ and $m_{(1,1)}(\cdot)$ on a set $M \subset \mathcal{P}_{4} / \Pi_{(1,1)}$ is not sufficient for $M$ to be relatively compact.

Lemma 3.10. Let $n \in \mathbb{N}, r \in \mathcal{D}_{n}$ and let $U$ be a complete set of representatives for $G_{r} / \Pi_{r}$. Then, for any $C>0$ and any matrix norm $\|\cdot\|: M(2 n ; \mathbb{R}) \rightarrow[0, \infty)$ there are only finitely many $G \in U$ with $\left\|{ }^{t} G^{-1} J G^{-1}\right\| \leq C$.

Proof. Firstly, for any $G, H \in U$ with $G \neq H$ we have ${ }^{\mathrm{t}} G^{-1} J G^{-1} \neq \pm{ }^{\mathrm{t}} H^{-1} J H^{-1}$. For if this were not the case, we would have $H^{-1} G=P \in \widetilde{\mathrm{Sp}}(2 n ; \mathbb{R})$, that is $[G]=[H P]=[H] \in G_{r} / \Pi_{r}$ which would be a contradiction. Secondly, if $G \in U$, then the entries of ${ }^{t} G^{-1} J G^{-1}$ are elements of $\frac{1}{r_{n}^{2}} \mathbb{Z}$. Since $M(2 n ; \mathbb{R})$ is a finite dimensional vector space, all norms are equivalent and we can choose a particular one. Let $\|\cdot\|$ be the maximum norm $\|G\|=\max \left\{\left|G_{i, j}\right| \mid 1 \leq i, j \leq 2 n\right\}$. Then $\left\|{ }^{\mathrm{t}} G^{-1} J G^{-1}\right\| \geq r_{n}^{-2}$ and by the above, $\left\|{ }^{\mathrm{t}} G^{-1} J G^{-1}-{ }^{\mathrm{t}} H^{-1} J H^{-1}\right\| \geq r_{n}^{-2}$ for all $G, H \in U$ with $G \neq H$. The lemma's statement now follows from the fact that the closed norm ball $\{G \in M(2 n ; R) \mid\|G\| \leq C\}$ is compact. 


\section{Notation and Remarks 3.11.}

(i) By the spectral theorem for symmetric matrices the eigenvalues of any matrix $Y \in \mathcal{P}_{n}$ are real and positive. We introduce functions $\lambda_{1}, \ldots, \lambda_{n}: \mathcal{P}_{n} \rightarrow$ $(0, \infty)$ such that $\lambda_{k}(Y)$ is an eigenvalue of $Y$ and $\lambda_{1} \leq \lambda_{2} \leq \cdots \leq \lambda_{n}$. Note that the $\lambda_{k}, 1 \leq k \leq n$, are continuous functions (see, e.g., Zed65, Theorem 1]).

(ii) We furthermore define functions $s_{1}, \ldots, s_{n}: \mathrm{GL}(n ; \mathbb{R}) \rightarrow(0, \infty)$ such that $s_{k}(G), 1 \leq k \leq n$, are the singular values of $G$ and $s_{1} \geq s_{2} \geq \cdots \geq s_{n}$. These functions satisfy $s_{k}(G)=\sqrt{\lambda_{n-k+1}\left({ }^{\mathrm{t}} G G\right)}$ which means in particular that they are continuous. Care has to be taken though as the $s_{k}$ are in general not invariant under conjugation. If, however, ${ }^{\mathrm{t}} G G \sim A \in \mathcal{P}_{n}$, then $s_{k}(G)$, $1 \leq k \leq n$, are the square roots of the eigenvalues of $A$.

(iii) We want to relate the functions $d_{j}: \mathcal{P}_{2 n} \rightarrow \mathbb{R}, 1 \leq j \leq n$, from Definition 3.1 to the singular values $s_{k}: \mathrm{GL}(2 n ; \mathbb{R}) \rightarrow(0, \infty), 1 \leq k \leq 2 n$. For any $G \in$ $\mathrm{GL}(2 n ; \mathbb{R})$ we have

$$
\begin{aligned}
{ }^{\mathrm{t}}\left({ }^{\mathrm{t}} G^{-1} J G^{-1}\right)\left({ }^{\mathrm{t}} G^{-1} J G^{-1}\right) & =-{ }^{\mathrm{t}} G^{-1} J G^{-1 \mathrm{t}} G^{-1} J G^{-1} \\
& \sim-G^{-1 \mathrm{t}} G^{-1} J G^{-1}{ }^{\mathrm{t}} G^{-1} J=-\left(\left({ }^{\mathrm{t}} G G\right)^{-1} J\right)^{2},
\end{aligned}
$$

which implies

$$
d_{j}\left({ }^{\mathrm{t}} G G\right)=s_{k}\left({ }^{\mathrm{t}} G^{-1} J G^{-1}\right)
$$

for $k \in\{2 n-2 j+2,2 n-2 j+1\}$.

The following lemma establishes the key matrix inequality required in our main theorem below.

Lemma 3.12. The inequality

$$
d_{n}\left({ }^{\mathrm{t}} G G\right)\left(\lambda_{2 n}(Y)\right)^{-1} \leq d_{n}(Y[G])
$$

holds for all $Y \in \mathcal{P}_{2 n}$ and $G \in \mathrm{GL}(2 n ; \mathbb{R})$.

Proof. By [Bha96, p. 72, (III.20)] one has

$$
\prod_{j=1}^{k} s_{i_{j}}(A) \prod_{j=1}^{k} s_{2 n-i_{j}+1}(B) \leq \prod_{j=1}^{k} s_{j}(A B)
$$

for all $A, B \in M(2 n ; \mathbb{R})$ and all $1 \leq i_{1}<\cdots<i_{k} \leq 2 n$. We choose $k=1, i_{1}=2 n$, $A=Y^{-1 / 2}$ and $B={ }^{\mathrm{t}} G^{-1} J G^{-1} Y^{-1 / 2}$ and obtain

$$
s_{2 n}\left(Y^{-1 / 2}\right) s_{1}\left({ }^{\mathrm{t}} G^{-1} J G^{-1} Y^{-1 / 2}\right) \leq s_{1}\left(Y^{-1 / 2 \mathrm{t}} G^{-1} J G^{-1} Y^{-1 / 2}\right) .
$$

We apply (3.2) again to the second factor of the left hand side of this inequality, this time with $A={ }^{\mathrm{t}} G^{-1} J G^{-1}, B=Y^{-1 / 2}, k=1$ and $i_{1}=1$, which yields

$$
\left(s_{2 n}\left(Y^{-1 / 2}\right)\right)^{2} s_{1}\left({ }^{\mathrm{t}} G^{-1} J G^{-1}\right) \leq s_{1}\left(Y^{-1 / 2}{ }^{\mathrm{t}} G^{-1} J G^{-1} Y^{-1 / 2}\right) .
$$

Now $\left(s_{2 n}\left(Y^{-1 / 2}\right)\right)^{2}=s_{2 n}\left(Y^{-1}\right)=\lambda_{1}\left(Y^{-1}\right)=\left(\lambda_{2 n}(Y)\right)^{-1}$. Furthermore, one has $s_{1}\left({ }^{\mathrm{t}} G^{-1} J G^{-1}\right)=d_{n}\left({ }^{\mathrm{t}} G G\right)$ by Remark 3.11(iii). Together, this shows that the left hand side of (3.3) matches the left hand side of the inequality in the statement of 
the lemma. We have a look at the right hand side of (3.3). Let $H:=Y^{1 / 2} G$. By Remark 3.11(iii) we have

$$
\begin{gathered}
s_{1}\left(Y^{-1 / 2}{ }^{\mathrm{t}} G^{-1} J G^{-1} Y^{-1 / 2}\right)=s_{1}\left({ }^{\mathrm{t}} H^{-1} J H^{-1}\right)=d_{n}\left({ }^{\mathrm{t}} H H\right)=d_{n}\left({ }^{\mathrm{t}} G Y^{1 / 2} Y^{1 / 2} G\right) \\
=d_{n}(Y[G]),
\end{gathered}
$$

which finishes the proof of the stated inequality.

Theorem 3.13. Let $M \subset \mathcal{P}_{2 n} / \Pi_{r}$. Assume that there are positive constants $C_{0}, C_{1}$ and $C_{2}$ such that $m_{r}([Y]) \geq C_{0}$, $\operatorname{det}([Y]) \leq C_{1}$ and $d_{n}([Y]) \leq C_{2}$ for all $[Y] \in M$. Then, $M$ has compact closure in $\mathcal{P}_{2 n} / \Pi_{r}$.

Proof. We denote by $\pi_{r}: \mathcal{P}_{2 n} \rightarrow \mathcal{P}_{2 n} / G_{r}, p_{r}: \mathcal{P}_{2 n} \rightarrow \mathcal{P}_{2 n} / \Pi_{r}$ and $\eta_{r}: \mathcal{P}_{2 n} / \Pi_{r} \rightarrow$ $\mathcal{P}_{2 n} / G_{r}$ the canonical projections. Note that $\eta_{r} \circ p_{r}=\pi_{r}$.

By Corollary 3.8, the set $\eta_{r}(M)$ is precompact. There is thus a precompact set $K \subset \mathscr{M}_{2 n, r}$, where $\mathscr{M}_{2 n, r}$ is the fundamental domain for $\mathcal{P}_{2 n} / \Pi_{r}$ defined by (3.1), with $\pi_{r}(K)=\eta_{r}(M)$. Consequently, one has

$$
M \subseteq \bigcup_{G \in U} p_{r}\left({ }^{\mathrm{t}} G K G\right),
$$

where $U$ is a full set of representatives of $G_{r} / \Pi_{r}$. The function $\lambda_{2 n}$ is continuous and therefore takes its maximum $\bar{M}>0$ on the closure $\bar{K}$ of $K$. By Lemma 3.12 one has

$$
d_{n}\left({ }^{\mathrm{t}} G Y G\right) \geq\left(\lambda_{2 n}(Y)\right)^{-1} d_{n}\left({ }^{\mathrm{t}} G G\right) \geq \bar{M}^{-1} d_{n}\left({ }^{\mathrm{t}} G G\right) \text { for all } Y \in K, G \in U .
$$

Let $\|\cdot\|_{2}$ be the spectral norm, that is, $\|G\|_{2}=s_{1}(G)$. Also, recall that $d_{n}\left({ }^{\mathrm{t}} G G\right)=$ $s_{1}\left({ }^{\mathrm{t}} G^{-1} J G^{-1}\right)$ by Remark 3.11(iii). Then, by Lemma 3.10 there are only finitely many $G \in U$ with $d_{n}\left({ }^{\mathrm{t}} G G\right)=s_{1}\left({ }^{\mathrm{t}} G^{-1} J G^{-1}\right)=\left\|{ }^{\mathrm{t}} G^{-1} J G^{-1}\right\|_{2} \leq C_{2} \bar{M}$. Let $G_{1}, \ldots, G_{N}$ be those $G \in U$. Because of inequality (3.5) we have

$$
d_{n}\left({ }^{\mathrm{t}} G Y G\right)>C_{2} \text { for all } Y \in K \text { and all } G \in U \backslash\left\{G_{1}, \ldots, G_{N}\right\},
$$

which in turn implies, by the assumption on $d_{n \mid M}$, that

$$
M \subseteq \bigcup_{j=1}^{N} p_{r}\left({ }^{\mathrm{t}} G_{j} K G_{j}\right) .
$$

The right hand side is a finite union of precompact sets and hence precompact. The set $M$ has thus compact closure, as claimed.

We can now characterise the relatively compact sets of the moduli spaces $\mathcal{M}_{n}^{r}$ and $\mathcal{M}_{n}^{r, H T}$.

Corollary 3.14. Let $n \in \mathbb{N}, r \in \mathcal{D}_{n}$ and $M \subset \mathcal{M}_{n}^{r}$. Assume that there are positive constants $C_{0}, C_{1}, C_{2}>0$ and a compact interval $I \subset(0, \infty)$ such that $g \in I$, $m_{r}(h) \geq C_{0}, \operatorname{det}(h) \leq C_{1}$ and $d_{n}(h) \leq C_{2}$ for all $[(h, g)] \in M$. Then $M$ has compact closure.

Corollary 3.15. Let $n \in \mathbb{N}$ and $r \in \mathcal{D}_{n}$. Then any set $M \subset \mathcal{M}_{n}^{r, H T}$ for which there exists a constant $C_{0}>0$ and a compact interval $I \subset(0, \infty)$ such that $m_{r}([h]) \geq C_{0}$ and $g \in I$ for all $[(h, g)] \in M$ is relatively compact. 
Proof. Let $M \subset \mathcal{M}_{n}^{r, H T}$ be a set for which there exist constants as stated. Any $[(h, g)] \in \mathcal{M}_{n}^{r, H T}$ satisfies $d_{k}(h)=g^{-1 / 2}$ for all $1 \leq k \leq n$ and hence $\operatorname{det} h=$ $\operatorname{det}\left(J^{-1} h\right)=g^{n}$. It follows from the last corollary that $M$ has compact closure in $\mathcal{M}_{n}^{r}$. By Corollary 3.3, $\mathcal{M}_{n}^{r, H T}$ is a closed subspace of $\mathcal{M}_{n}^{r}$. Hence, the closure of $M$ is still contained in $\mathcal{M}_{n}^{r, H T}$.

Remark 3.16. Let $\left(\Gamma^{r} \backslash H_{n}, \mathbf{m}\right)$ be a normalised Heisenberg manifold with $\mathbf{m}=$ $(h, g)$. By (2.6) and (2.5) we have the following inequality for the sectional curvatures $K$,

$$
K \leq g^{-1} d_{n}^{2}(h) .
$$

Hence one can equivalently replace the uniform bound on $d_{n}$ in Corollary 3.14 by a uniform upper bound on the sectional curvatures.

\section{REFERENCES}

[Bha96] R. Bнатia Matrix analysis. Springer New York, 1996.

[Bol18] S. Boldt The height of compact nonsingular Heisenberg-like Nilmanifolds. Dessertation, Doi: $10.18452 / 18924,2018$.

[Ebe94] P. Eberlein Geometry of 2-step nilpotent groups with a left invariant metric. Ann. Sci. École Norm. Sup. (4) 27, no. 5, 1994, 611-660.

[GL87] P.M. Gruber and C.G. LekKerkerker Geometry of numbers. 2nd ed. North Holland, 1987.

[GW86] C. S. Gordon And E. N. Wilson The spectrum of the Laplacian on Riemannian Heisenberg manifolds. Mich. Math. J., 33, 1986, 253-271.

[Tas20] K. TASHIRO Precompactness theorem for compact Heisenberg manifolds with sub-Riemannian metrics and the Gromov-Hausdorff topology. arXiv preprint. arXiv:2004.09407, 2020.

[Ter88] A. TerRas Harmonic analysis on symmetric spaces and applications. II. Springer New York, 1988.

[Zed65] M. Zedek Continuity and location of zeros of linear combinations of polynomials. Proc. Am. Math. Soc., 16, 1965, 78-84.

Email address: boldt@math.uni-leipzig.de

Mathematisches Institut, Universität Leipzig, 04081 Leipzig, Germany 